\title{
¿Quién gobierna América del Norte? Elites, redes y organizaciones
}

\author{
Carlos Alba VegA*
}

Salas-Porras, Alejandra y Matilde Luna, coords., ¿Quién gobierna América del Norte? Elites, redes y organizaciones, México, unAm, 2012, 336 pp.

Este libro es producto de un esfuerzo colectivo, sin duda, resultado de la experiencia y del trabajo de mucho tiempo por parte de los autores. Entre sus principales aciertos habrá que reconocer la novedad de los temas tratados; el esfuerzo comparativo en algunos de ellos; la voluntad de indagar más sobre diversos aspectos de Estados Unidos y Canadá para conocer mejor el conjunto de la zona, y México dentro de ella; la unidad que se consigue en el conjunto, a pesar de la diversidad de temas y de enfoques; la actualidad de los problemas que aborda; el carácter interdisciplinario de quienes participaron en los estudios; el equilibrio entre los desarrollos teóricos y metodológicos de cada trabajo; la mirada crítica de las ciencias sociales, que buscan develar lo que se presenta encubierto.

En efecto, varios de los asuntos tratados en el libro han sido abordados por muy pocos investigadores: el enfoque comparativo de las elites de los tres países de América del Norte y de su participación en la construcción del Tratado de Libre Comercio de América del Norte (TLCAN), emprendido por Ricardo Tirado; el texto de Teresina Gutiérrez-Haces que se centra de manera especial en Canadá, un país menos conocido y estudiado por los mexicanos; el estudio de Alejandra Salas-Porras sobre los think tanks o centros de pensamiento como actores que influyen en las políticas públicas; la Comisión Trilateral y el Grupo Mexicano, abordados por Matilde Luna y José Luis Velasco como parte de las elites

\footnotetext{
*Centro de Estudios Internacionales, El Colegio de México, calba@colmex.mx.
} 
El libro se orienta a analizar algunos temas y problemas contemporáneos derivados de la reinserción de México a la economía mundial, o más especificamente, a la zona de América del Norte. económicas y políticas del TLCAN; las asociaciones de profesionistas y su papel en la liberación de los servicios profesionales, analizadas por María de Lourdes y María del Socorro Marquina Sánchez y Alejandro Arnulfo Ruiz León; la migración calificada o, si se quiere, "la fuga de cerebros" de México, examinada por Camelia Tigau; los estudios orientados a ahondar más en la problemática de la ciencia, la tecnología y la innovación en México, como son los casos de los biocombustibles, explorados por Edit Antal, y la investigación de Kenneth Shadlen sobre el régimen de patentes que otorgan derechos de exclusión limitados a los propietarios del conocimiento.

El hilo conductor que engarza los capítulos es el estudio de las elites, ya sean económicas, políticas o profesionales, que por medio de sus organizaciones han participado en la reorientación del modelo de desarrollo en México. Este modelo, que emerge después de la crisis de la deuda de 1982, sirve de telón de fondo para examinar en el libro el papel que han desempeñado ciertos actores económicos y políticos: los que están colocados en el vértice de la pirámide. Por lo tanto, los actores sociales, las clases populares, los movimientos sociales, las organizaciones de la sociedad civil, los miembros que no forman parte de las elites, todos estos actores subalternos en América del Norte son un tema pendiente cuyo estudio sugiere la lectura de esta publicación. Porque existe ahora un libro que se interroga sobre las elites que gobiernan América del Norte, hace falta otro que aborde diversos actores de la globalización desde abajo, que participan con sus propias organizaciones y redes en la región: los migrantes, los pequeños empresarios y comerciantes, las comunidades transnacionales, los sindicatos, los movimientos sociales.

El libro se orienta a analizar algunos temas y problemas contemporáneos derivados de la reinserción de México a la economía mundial, o más específicamente, a la zona de América del Norte. Hoy, México es considerado miembro de esta región por razones que van más allá de las geográficas. A lo largo del tiempo y sobre todo en las últimas décadas, se ha ido tejiendo una densa relación entre México y Estados Unidos y entre este país y Canadá; una intensa red de relaciones que se han formalizado desde finales de los años ochenta y principios de los noventa en un Tratado de Libre Comercio de América del Norte.

Ricardo Tirado muestra claramente en su texto que tanto Canadá como México cambiaron su política nacionalista y proteccionista a causa de su debilidad provocada por la crisis de principios de los ochenta y de 
la caída de los precios del petróleo que afectó sus economías, y advierte que la decisión de crear el TLCAN se fraguó en Estados Unidos para acceder a ese energético y utilizar las ventajas de la zona y así mejorar su productividad y sus exportaciones en el contexto de la globalización. Para probarlo, Tirado aprovecha a fondo sus investigaciones sobre las elites empresariales de los tres países, sobre todo de México y Estados Unidos, gracias a lo cual analiza cómo se llegó a la decisión del TLCAN en cada uno de ellos. Muestra que la interrelación entre las elites económicas, políticas y del conocimiento en Estados Unidos está fuertemente institucionalizada a través de una densa red de personalidades, organizaciones gremiales, think tanks y empresas privadas que influyen de manera importante en la política comercial de ese país. Sería el sector empresarial, constituido por las grandes empresas el que, a diferencia de los de México y Canadá, logró, con el apoyo del gobierno y casi sin ninguna disputa, llevar al resto de los empresarios al proyecto del Tratado de Libre Comercio de América del Norte.

El estudio de las elites empresariales de Canadá es una de las especialidades de Teresina Gutiérrez Haces. En su artículo muestra la importancia que tuvieron en el cambio económico tanto la crisis de 1982 como la creación de la Real Comisión McDonald, encargada por el gobierno federal de analizar el modelo de desarrollo. Destaca dos conclusiones importantes de esta agrupación: era necesario reajustar la política económica internacional y reconsiderar la relación con Estados Unidos. En este contexto, las elites empresariales se unificaron y organizaron para promover sus intereses mediante las políticas públicas, la política comercial internacional y hasta por medio de su influencia en la determinación del perfil que debían tener los futuros primeros ministros de Canadá y los miembros del gabinete. Así, a partir de 1984 se genera una identidad entre los intereses y las políticas de los gobiernos canadienses y las elites empresariales y sus organizaciones, cuya fuerza proviene del alto grado de articulación que han logrado las más poderosas y transnacionalizadas y de los consensos que consiguieron en la comunidad de negocios.

Estas elites empresariales canadienses han logrado cambiar las políticas públicas y las leyes de Canadá; por ejemplo, lograron desarticular el Programa Nacional de Energía, crear la Nueva Ley de Competencia afín a sus intereses, promover la firma del Tratado de Libre Comercio de Canadá con Estados Unidos en 1988 y el TLCAN en 1993, participar con grupos de expertos para representar sus intereses en las políticas que

La interrelación entre las elites económicas, políticas y del conocimiento en Estados Unidos influye de manera importante en la política comercial de ese país.

Las elites empresariales canadienses han logrado cambiar las políticas públicas y las leyes de Canadá. 
Alejandra Salas-Porras examina el papel que desempeñaron

los centros de pensamiento o think tanks. Su trabajo muestra cómo se ha construido esa red de centros de pensamiento a favor del TLCAN. emergen de los organismos económicos internacionales como la Organización Mundial de Comercio (OMC) y la Organización para la Cooperación y el Desarrollo Económicos (OCDE).

Tirado expone las ideas medulares que llevaron a las elites económicas y políticas de México al cambio de modelo de desarrollo que se coronó con el TLCAN. El poder autoritario del régimen emprendió un conjunto de reformas económicas que fueron favorables a los intereses de las elites empresariales. La débil oposición a estas reformas fue enfrentada por medio de mecanismos corporativos y clientelares tanto entre los empresarios opositores como entre los trabajadores. En cambio, en Estados Unidos y Canadá sí hubo una oposición de envergadura que, si bien no logró bloquear la firma del TLCAN, consiguió que se incorporaran acuerdos paralelos en materia laboral y ambiental.

Alejandra Salas-Porras examina el papel que desempeñaron los centros de pensamiento o think tanks en el lanzamiento de la idea, en la creación de una opinión pública favorable, en la formación de la coalición política comprometida con la integración económica a través del TLCAN y en su defensa frente a los ataques de los grupos proteccionistas y aislacionistas que en Estados Unidos nunca se han debilitado, como sí ha ocurrido en México y Canadá.

Su trabajo muestra cómo se ha construido esa red de centros de pensamiento a favor del TLCAN, la cual patrocina equipos de investigación y grupos de trabajo, publica artículos y libros que influyen en los medios académicos y periodísticos; organiza diversos tipos de actividades públicas y privadas con líderes de opinión y políticos que tienen presencia en los medios de comunicación, en especial con los legisladores del Congreso de Estados Unidos. Para Salas-Porras, la influencia de las elites en esa red es abrumadora porque combina las elites estatales, corporativas, académicas e intelectuales que interactúan en las juntas de gobierno y en las actividades de los centros de pensamiento. Es decir, participan en la formación y desarrollo de una comunidad epistémico, que articula y coordina una constelación de intereses diversificados que han construido el imaginario de la región con una visión liberal y aperturista en el plano económico, y controla el conocimiento y los espacios en que se toman las decisiones sobre el comercio y la política exterior en el ámbito de la región de América del Norte.

Desde otro ángulo, Matilde Luna y José Luis Velasco indagan sobre una organización importante y poco conocida que se articula en torno al 
TLCAN: la Comisión Trilateral y, dentro de ella, examinan al Grupo Mexicano. Su estudio tiene dos intereses principales: por una parte, identificar las bases de autoridad de estos organismos, es decir, los mecanismos institucionales y los recursos (relacionales, económicos, políticos y simbólicos) que contribuyen a transformar el poder económico y político de las elites en autoridad; y por la otra, analizar la tensión entre la eficacia y la legitimidad.

En este texto resulta tan importante el esfuerzo de conceptualización teórica y el enfoque metodológico como el trabajo empírico. Su pregunta central busca explicar las bases de la autoridad, es decir, el poder y la legitimidad, de los sistemas asociativos complejos que participan como parte de las estructuras de gobernanza global, como la Comisión Trilateral y el Grupo México, y su grado de representatividad y de eficacia.

Los autores concluyen que en estas estructuras de gobernanza global, la representatividad de los sistemas asociativos complejos es imprecisa, ambigua y múltiple; y que para superar tales problemas, estos sistemas necesitan satisfacer algunos principios.

En lo que concierne a la eficacia de los sistemas asociativos complejos ocurriría lo mismo: para ser eficaces, éstos necesitan lograr sus propios objetivos colectivos y, al mismo tiempo, brindar beneficios concretos a sus miembros, mantener vigente la organización y contribuir a la vida asociativa global.

A partir de este marco, Luna y Velasco sostienen que la legitimidad del Grupo Mexicano es precaria porque existe una fuerte ambigüedad entre los intereses nacionales y los internacionales, y entre las preocupaciones de índole económica (la lógica de la ganancia) y política (la lógica del poder). El alto grado de autonomía individual de los miembros hace posible que el sistema privilegie los intereses particulares sobre los intereses más generales de la sociedad mexicana.

En conclusión, el equipo mexicano adolece de un problema que enfrentan los sistemas asociativos complejos: la dificultad de mantener un equilibrio entre cohesión, dispersión, logros prácticos y la producción de capital organizativo que permita integrar a otros actores y puntos de vista. Para los autores, el Grupo Mexicano es muy compacto y sus miembros controlan o representan intereses de diversas fuentes de poder económico, político, relacional e informacional. Sus densas relaciones con grandes empresas y organizaciones mexicanas poderosas que operan en el ámbito regional y estadunidense permiten suponer que desempeñan
Los autores

concluyen que en estas estructuras de gobernanza global la representatividad de los sistemas asociativos complejos es imprecisa, ambigua y múltiple. 
En la primera

parte destacan

la importancia

de los servicios

profesionales en las

economías

globalizadas, para

después identificar algunos colegios de

profesionistas

como una elite que influye en la toma

de decisiones

relacionadas

con el comercio

de servicios

transfronterizos. un papel clave en el diseño de la política nacional. Por lo tanto, esas características del Grupo Mexicano, que parecen suficientes para una agrupación con fines privados, resultan insuficientes para una estructura de gobernanza global que aspira a representar a un amplio sector de la opinión pública mundial. De ahí que si bien el Grupo México es influyente y cuenta con mucho poder, tiene un déficit de legitimidad porque es poco representativo.

El trabajo de María de Lourdes y María del Socorro Marquina Sánchez y Alejandro Arnulfo Ruiz León analiza la participación de los abogados y los ingenieros mexicanos en el comercio de servicios profesionales dentro del TLCAN. La pregunta principal que guía al trabajo es ¿por qué y cómo sólo ciertos grupos de abogados e ingenieros pudieron influir en las negociaciones de los servicios profesionales en el TLCAN?

En la primera parte destacan la importancia de los servicios profesionales en las economías globalizadas, para después identificar algunos colegios de profesionistas como una elite que influye en la toma de decisiones relacionadas con el comercio de servicios transfronterizos. Después de examinar a los profesionistas como parte de las elites y de mostrar la importancia que tiene el sector terciario en la globalización, los investigadores analizan el proceso de negociación y las controversias para liberalizar el comercio transfronterizo de los abogados y los ingenieros. Argumentan que los profesionistas necesitan de la organización gremial para influir en las decisiones relacionadas con el ejercicio de su profesión, y que esa capacidad de influencia depende tanto de recursos de poder como del marco socioinstitucional en el que desarrollan su acción. Según su estudio, el hecho de que en Canadá y Estados Unidos la pertenencia no es libre como en México, sino obligatoria, y que además es necesario contar con una experiencia profesional mínima antes de obtener la licencia, otorga a estas organizaciones la capacidad de determinar la calidad educativa y de tener un control oligopólico sobre el ejercicio de la profesión. Mientras en México las cédulas profesionales son otorgadas por el Estado, las asociaciones de aquellos países expiden las licencias (cédulas) para el ejercicio profesional, las cuales son renovadas periódicamente por medio de actualizaciones, exámenes y seguros para protección de los usuarios, con lo que pueden controlar el mercado de los servicios profesionales. Esa diferencia en la certificación, sin embargo, constituye una barrera para el acceso de los abogados e ingenieros mexicanos que desean prestar sus servicios en América del Norte. 
Camelia Tigau estudia la diáspora de profesionistas mexicanos en Norteamérica y se pregunta si disponen de capacidades de organización, heterogeneidad y posicionamiento profesional para contribuir, en su calidad de elites, al desarrollo de México. Su hipótesis es que para promover incentivos orientados a que una parte de la diáspora de profesionistas regrese o se organice en favor del desarrollo de México, el Estado tendría que competir con otros actores fuertes, en especial con las empresas transnacionales, ya que, según este estudio, son los principales empleadores de esta fuerza de trabajo calificada. La autora concluye que México no cuenta con una diáspora suficientemente posicionada y organizada, como lo serían los casos de India y China. Además, existen ciertas contradicciones y preguntas cruciales sobre este tema, por ejemplo, si el desarrollo científico y tecnológico de un país debe fincarse en el aprovechamiento de los recursos de su diáspora calificada, más que en sus recursos internos. También habría que considerar la actitud de la diáspora hacia el gobierno de su país.

Además de estos factores, Tigau señala diversos obstáculos que dificultan la repatriación de miembros de la diáspora o de sus recursos, tales como la falta de incentivos de retorno suficientemente atractivos, la escasez de oportunidades laborales, la inseguridad, los fuertes atractivos para emigrar al Norte por los incentivos que se presentan en el marco del TLCAN, la competencia que enfrentaría el gobierno ante las ofertas de las empresas transnacionales. En este contexto, la autora sugiere que para el gobierno la atracción de fuerza de trabajo calificada debería ser una prioridad, como lo es para otros países y como es para México la atracción de inversión extranjera directa.

Edit Antal analiza el caso de las políticas públicas de los biocombustibles en México para ilustrar el problema de la gobernanza en ciencia y tecnología en el ámbito de la zona del TLCAN. La autora advierte que la gobernanza de los biocombustibles es un verdadero desafío porque se trata de desarrollar nuevos productos, en parte promovidos en el marco de los acuerdos internacionales en torno al cambio climático, que suponen la creación también de un nuevo sector económico, la generación de nuevas tecnologías y la coordinación de actividades agrícolas, industriales y de servicios. Un obstáculo adicional sería la ambigüedad legal en torno a la comercialización del biodiesel y la oposición de Pemex al uso de este tipo de carburantes. Para la autora, estas actividades de coordinación deben ser responsabilidad no sólo de las elites del sector privado
Camelia Tigau sugiere que para el gobierno la atracción de fuerza de trabajo calificada debería ser una prioridad, como lo es para otros países y como lo es para México la atracción de inversión extranjera directa. 
Kenneth Shadlen muestra cómo

México adoptó, antes de la firma

del TLCAN,

un régimen de patentes extremadamente "fuerte" que otorga derechos desproporcionados a los dueños del conocimiento en comparación con los usuarios del conocimiento. guiadas de manera individual con la brújula del mercado y con miras a la exportación hacia Estados Unidos, sino también del Estado, para que esta actividad pueda orientarse también a promover el crecimiento y el desarrollo del sector rural del país. Antal advierte que el mayor problema para la gobernanza en el caso de biocombustibles como el etanol, es que no son rentables en el mercado, ya que sus costos y precios son más altos que los de los bienes que pretende sustituir, por lo que se necesita apoyar su producción con recursos públicos, sea mediante la vía fiscal o de subsidios directos a los productores de los insumos. Desde su perspectiva hacen falta estudios previos más allá de los estudios técnicos de viabilidad que la Secretaría de Energía ha confiado al Banco Interamericano de Desarrollo y a la Agencia de Cooperación Técnica de Alemania. Esos estudios necesitarían considerar también los costos ambientales, sociales y económicos, así como la balanza bioenergética de los combustibles. Para Edit Antal, una gobernanza exitosa de los biocombustibles necesitaría enmarcarse en un plan de desarrollo económico y social integral, con recursos propios y canales abiertos y transparentes.

Kenneth Shadlen se interesa en averiguar por qué las elites mexicanas han sido incapaces de crear un proyecto nacional con políticas activas en ciencia, tecnología e innovación, es decir, orientado a promover el desarrollo de una economía basada en el conocimiento. Para responder a esta pregunta, examina las coaliciones políticas que actúan en las áreas de propiedad intelectual y en las políticas de innovación. Sobre la propiedad intelectual, Shadlen analiza las patentes que otorgan derechos de exclusión a los propietarios del conocimiento, un recurso intangible, inagotable y no concurrente. Muestra cómo México adoptó, antes de la firma del TLCAN, un régimen de patentes extremadamente "fuerte" que otorga derechos desproporcionados a los dueños del conocimiento en comparación con los usuarios del conocimiento; es decir, una amplia gama de tipos de conocimiento, con un control extensivo sobre el uso de conocimiento privado a favor de los propietarios versus los derechos de los usuarios, y por un tiempo más largo. De eso resultó un incremento de los costos de los bienes intensivos en tecnología y el aumento de las barreras para acceder al conocimiento, sin obtener los beneficios de alentar la investigación científica, tecnológica y la innovación. Ese régimen fuerte, que no correspondía al nivel de capacidades científicas, tecnológicas y de innovación de México, tuvo lugar en el marco de la liberalización e internacionalización de la economía que propició cambios en la estructura 
industrial, los cuales alteraron el tamaño y la forma de las coaliciones que presionan por diferentes tipos de política en cuanto a patentes e innovación; eso fortaleció una coalición de elites que promueve una forma de integración en la economía global de baja tecnología y que al mismo tiempo inhibe y aísla a otras coaliciones científicas y del gobierno que podrían pugnar por un proyecto alternativo.

\section{Conclusiones}

En campos de estudio distintos, todas las autoras y autores convergen en varios aspectos que fortalecen la unidad del libro. Se preguntan si existe una elite regional que gobierna la región de América del Norte en el marco del TLCAN, quiénes la constituyen, cómo se construyó y a través de qué organizaciones ejerce el poder.

El TLCAN es atribuible ante todo a las elites empresarial y gubernamental de Estados Unidos, las cuales están íntimamente interrelacionadas a través de personalidades, organizaciones, gremios, think tanks y empresas que estaban interesadas en mejorar el posicionamiento productivo y de exportación de su país, así como acceder a los energéticos de sus vecinos.

Un grupo de think tanks, constituidos por elites estatales, corporativas, académicas e intelectuales convertidas en una comunidad epistémica que controla el conocimiento, logró construir una red a favor del TLCAN mediante las actividades que promueve, como son la formación de equipos de investigación, grupos de trabajo, publicaciones y programas en los medios de comunicación de masas, organización de eventos públicos y privados con líderes de opinión y miembros de la clase política que toman decisiones.

El Grupo México, siendo miembro de un sistema asociativo complejo como lo es la Comisión Trilateral, participa con autoridad en la gobernanza global que enmarca al TLCAN por medio de los mecanismos institucionales y las diversas fuentes de recursos económicos, sociales, políticos y simbólicos que posee, pero no cuenta con legitimidad por la ambigüedad de los intereses nacionales e internacionales que representa y las distintas lógicas económicas (la ganancia) y políticas (el poder) que lo guían.

Paralelamente a estos intereses empresariales y gubernamentales, se ha venido generando una interrelación entre México y Estados Unidos
Un grupo

de think tanks, constituidos por elites estatales, corporativas, académicas e intelectuales convertidas en una comunidad epistémica que controla el conocimiento, logró construir una red a favor del TLCAN. 
El proceso de integración y liberalización económica ha conducido a una concentración de la propiedad, el conocimiento,

la información y la tecnología, que son controlados por elites económicas, gubernamentales y del conocimiento. que se expresa en los campos sociales, económicos y políticos: inversión extranjera directa, industrias maquiladoras de exportación, turismo, migraciones, remesas y contrabando de norte a sur, capital fugado en sentido inverso, tráfico ilegal de narcóticos, deuda externa, comercio.

En Canadá, desde mediados de los ochenta, independientemente del partido en el gobierno federal o provincial, se produjo una identidad entre los intereses y las políticas de los gobiernos canadienses y las elites empresariales de este país, las que por su fuerte articulación en organizaciones corporativas y su transnacionalización, lograron cambiar las políticas públicas y las leyes de su país.

La crisis económica de 1982 y la caída de los precios del petróleo fueron el detonador de un cambio de modelo de desarrollo que fue impulsado por una parte de las elites políticas y empresariales, las cuales sustituyeron en Canadá y México el modelo nacionalista y proteccionista por otro volcado al exterior. Si en Estados Unidos y en Canadá estas elites lograron derrotar a las fuerzas opositoras al TLCAN a cambio de acuerdos paralelos en materia ambiental y laboral, en México no hubo nunca una fuerte oposición por la naturaleza del régimen autoritario y las formas corporativas y clientelares que se utilizaron.

El proceso de integración y liberalización económica ha conducido a una concentración de la propiedad, el conocimiento, la información y la tecnología, que son controlados por elites económicas, gubernamentales y del conocimiento, las que cuentan con distintos tipos de poder que ejercen a través grandes empresas transnacionales y nacionales, think tanks y diversos tipos de organizaciones e instituciones.

Dentro de los gremios profesionales, se observan también los problemas y asimetrías en las relaciones, que en el caso mexicano estuvieron dominadas por los economistas del TLCAN, y donde los colegios de abogados e ingenieros mexicanos encuentran severas dificultades para promover el comercio de servicios profesionales en Estados Unidos y Canadá. Cuando esos servicios de trabajo calificado mexicano se convierten en una diáspora de profesionistas establecida en Estados Unidos, su potencial económico y profesional resulta atractivo para reorientarlos a la promoción del desarrollo de su país de origen. Sin embargo, el Estado tendría que reorientar su política de inmigración de fuerza de trabajo calificada, ofrecer incentivos de retorno muy importantes para competir con quienes la emplean y ofrecer ventajas adicionales a las que obtienen en el extranjero. 
Otro problema de gobernanza, ahora en el campo de la ciencia y las nuevas tecnologías, es el de la política de biocombustibles, donde se presenta una ambigüedad legal y una tensión entre actores privados, gubernamentales y paraestatales en disputa por el nuevo combustible, por lo que resulta imprescindible emprender una política de Estado que ubique el energético en un plan integral de desarrollo que incluya al sector rural, el que más perdió en las negociaciones del TLCAN.

En el mismo campo de la ciencia, la tecnología y la innovación, las elites mexicanas no han logrado crear un proyecto nacional con políticas activas, orientado a promover el desarrollo de una economía basada en el conocimiento. En el ámbito de la propiedad intelectual, en cuanto a patentes, México ha otorgado amplios derechos de exclusión a los dueños del conocimiento privado en comparación con los usuarios de éste. Por lo tanto, las barreras y los costos de productos como los farmacéuticos son más elevados que en otros países comparables con México. Esto ha dependido también del poder de la coalición de las elites económicas y políticas y de las transformaciones industriales ocurridas en el marco de la liberalización y el TLCAN, las cuales inhiben la formación de otras coaliciones con proyectos alternativos.

Los grandes ausentes de este proceso de integración son los sectores populares, los pequeños empresarios, los sindicatos, las organizaciones de la sociedad civil y muchas otras asociaciones que no forman parte de las elites económicas y políticas. Por lo tanto, el diseño de las políticas públicas en México desde los años ochenta ha estado controlado por un grupo de la elite económica, tecnocrática y política que tiene fuertes vínculos con las grandes empresas nacionales y transnacionales.

En síntesis, la originalidad y la calidad de las investigaciones comentadas sumariamente nos permiten reconocer que estamos ante un libro fundamental para quienes se interesan en conocer más a fondo cómo participa México, y dentro él las elites y diversas redes y organizaciones, en esta zona económica del Norte. La amplitud de los temas y la magnitud de los problemas que se podrían tratar sobre América del Norte desbordan las posibilidades que ofrece un solo libro. Por eso, seguramente las coordinadoras dejaron fuera de la mira otros temas importantes que ya han sido tratados por otros autores, tales como las fronteras, la violencia, el narcotráfico, las migraciones.
Los grandes

ausentes

de este proceso de integración son los sectores populares, los pequeños empresarios, los sindicatos, las organizaciones de la sociedad civil y muchas otras asociaciones que no forman parte de las elites económicas y políticas. 
